\title{
Hadronic current correlation functions at finite temperature in the NJL model
}

\author{
R.L.S.Farias* and G. Kreint \\ Instituto de Física Teórica, Universidade Estadual Paulista \\ Rua Pamplona, 145, 01405-900, São Paulo, SP - Brazil \\ O.A.Battiste $\ddagger$ \\ Departamento de Física, Universidade Federal de Santa Maria \\ POBox 5093, 97119-900 Santa Maria, RS - Brazil
}

\begin{abstract}
Recently there have been suggestions that for a proper description of hadronic matter and hadronic correlation functions within the NJL model at finite density/temperature the parameters of the model should be taken density/temperature dependent. Here we show that qualitatively similar results can be obtained using a cutoff-independent regularization of the NJL model. In this regularization scheme one can express the divergent parts at finite density/temperature of the amplitudes in terms of their counterparts in vacuum.
\end{abstract}

\footnotetext{
*Electronic address: ricardof@ift.unesp.br

${ }^{\dagger}$ Electronic address: gkrein@ift.unesp.br

${ }^{\ddagger}$ Electronic address: orimar@ccne.ufsm.br
} 
Recently there have been suggestions that for a proper description of hadronic matter and hadronic correlation functions within the Nambu-Jona-Lasinio (NJL) model at finite densities and temperatures the parameters of the model should be taken temperature and density dependent. In a study of the color flavor locked phase of QCD within the NJL model Casalbuoni et al. [1] have shown that in order to get sensible results the ultraviolet cutoff of the model should increase with density. Similarly, Shakin et al. 22, 3] in studies of hadronic correlation functions found that the NJL model may have a broader range of application than previously considered if one allows a significant temperature dependence of the parameters of the model, in particular of the cutoff.

The aim of the present communication is to show that qualitatively similar results of Refs. [2] and [3] can be obtained using a cutoff-independent regularization of the NJL model [4, 5]. In this novel regularization scheme, one assumes an implicit regularization of the divergent integrals and by making use the scaling properties of these integrals, one can express the divergent parts of the amplitudes at finite temperature/density in terms of their counterparts at zero temperature/density. At one loop there are only two divergent integrals and by fitting these to physical quantities at zero temperature and density, no cutoff needs to be introduced. One consequence of this is that all momentum integrals over the Fermi-Dirac distributions can be extended to infinity, and need not be cutoff at some large mass scale. Here we apply this regularization scheme to study finite temperature hadronic current correlation functions [6].

At the level of the mean field (or one loop) approximation, there appear two divergent integrals, one quadratically divergent $\left(I_{q}\right)$ and one logaritmically divergent $\left(I_{l}\right)$ :

$$
I_{q}\left(M^{2}\right)=\int_{r e g} \frac{d^{4} k}{(2 \pi)^{4}} \frac{1}{\left(k^{2}-M^{2}\right)} \quad I_{l}\left(M^{2}\right)=\int_{r e g} \frac{d^{4} k}{(2 \pi)^{4}} \frac{1}{\left(k^{2}-M^{2}\right)^{2}}
$$

It is not difficult to show that these integrals satisfy the following scaling properties [4],

$$
\begin{aligned}
& I_{q}\left(M^{2}\right)=I_{q}\left(\mu^{2}\right)+\left(M^{2}-\mu^{2}\right) I_{l}\left(\mu^{2}\right)+\frac{i}{(4 \pi)^{2}}\left[M^{2}-\mu^{2}-M^{2} \log \left(M^{2} / \mu^{2}\right)\right] \\
& I_{l}\left(M^{2}\right)=I_{l}\left(\mu^{2}\right)-\frac{i}{(4 \pi)^{2}} \log \left(M^{2} / \mu^{2}\right)
\end{aligned}
$$

where $\mu$ is an arbitrary mass scale. In general, when dealing with finite density/temperature, the mass scale that appears in $I_{q}$ and $I_{l}$ is density/temperature dependent. The obvious use one can make from the scaling relations of Eqs. (2) and (3) is to express the divergent integrals at finite temperature in terms of the zero temperature integrals. Moreover, 
since the finite parts are integrated over all momenta, temperature effects coming from the Fermi-Dirac distribution function are not cutoff at some finite momentum as in cutoff regularization schemes. In this way, there is no need to use arbitrary temperature dependencies on the cutoffs [2] in order to extend the range of momentum integrals. In order to illustrate these properties, we consider the pseudoscalar correlation function at high temperatures. In particular, we will show how the regularization scheme naturally isolates the finite, temperature-dependent contributions from purely divergent, vacuum effects, and compare with the corresponding results using a cutoff scheme.

The pseudoscalar correlator $C_{P}\left(P^{2}, T\right)$ is the Fourier transform of the thermal average of the time-ordered product of pseudoscalar currents $j_{5}=\bar{\psi} \gamma_{5} \tau^{i} \psi$. The imaginary part of $C_{P}\left(P^{2}, T\right)$ can be written as [2]

$$
\operatorname{Im} C_{P}\left(P^{2}, T\right)=\frac{\operatorname{Im} \Pi_{P}\left(P^{2}, T\right)}{\left[1-2 G \operatorname{Re}_{P}\left(P^{2}, T\right)\right]^{2}+\left[2 G \operatorname{Im}_{P}\left(P^{2}, T\right)\right]^{2}}
$$

where $\Pi_{P}\left(P^{2}, T\right)$ is the pseudoscalar polarization function. In the frame $\vec{P}=0$, the imaginary part of $\Pi_{P}\left(P^{2}, T\right)$ is given by

$$
\operatorname{Im} \Pi_{P}\left(\omega^{2}, T\right)=\frac{N_{c} \omega}{4 \pi}\left(\omega^{2}-4 M^{* 2}\right)^{1 / 2}[1-2 n(\omega / 2)]
$$

where $M^{*}$ is the temperature-dependent constituent quark mass, and $n$ is the Fermi-Dirac distribution function. The real part of $\Pi_{P}\left(\omega^{2}, T\right)$ can be obtained by the dispersion integral, which turns out to be ultraviolet divergent. As announced previously, one can express the divergent part of the amplitude in terms of $I_{q}$ and $I_{l}$ :

$$
\operatorname{Re} \Pi_{P}\left(\omega^{2}, T\right)=\frac{N_{c}}{4 \pi^{2}} \mathcal{P} \int_{4 M^{* 2}}^{\infty} \frac{d s\left[\omega^{4}-2 s^{2} n(\sqrt{s} / 2)\right]}{s^{3 / 2}\left(s-4 M^{* 2}\right)^{1 / 2}}-4 N_{c} \omega^{2}\left[i I_{l}\left(4 M^{* 2}\right)-\frac{i I_{q}\left(4 M^{* 2}\right)}{2 \omega^{2}}\right]
$$

The principal value integral is finite. Using the scaling relations above, one can now reexpress $I_{l}\left(4 M^{* 2}\right)$ and $I_{q}\left(4 M^{* 2}\right)$ in terms of $I_{l}\left(M^{2}\right)$ and $I_{q}\left(M^{2}\right)$. These last two integrals are vacuum quantities and can be fitted to chiral parameters, like $\langle\bar{\psi} \psi\rangle$ and $f_{\pi}$.

In Figure 1 we plot the integrals

$$
F_{\text {cut }}\left(\omega^{2}, T\right)=\mathcal{P} \int_{4 M^{* 2}}^{\infty} d s \frac{\sqrt{s\left(s-4 M^{* 2}\right)}}{s-\omega^{2}} n(\sqrt{s} / 2) e^{-\left(s-4 M^{* 2}\right) / 4 \alpha^{2}(T)}
$$

and

$$
F_{i m p}\left(\omega^{2}, T\right)=\mathcal{P} \int_{4 M^{* 2}}^{\infty} d s \frac{\left[\omega^{4}-2 s^{2} n(\sqrt{s} / 2)\right]}{s^{3 / 2}\left(s-4 M^{* 2}\right)^{1 / 2}}
$$


where $\alpha(T)$ is the temperature-dependent cutoff. These functions are precisely the parts of the integrals that depend on the Fermi-Dirac distribution. While in $F_{c u t}$ the high energy region is cutoff by the form factor, $F_{i m p}$ is not - but its ultraviolet behavior is softened by the subtractions done in order to extract the infinite contributions. We use $T=1.5 T_{c}$, with $T_{c}=150 \mathrm{MeV}$, for which $M^{*}=10 \mathrm{MeV}$. The Figure shows that the effect of a temperature dependent cutoff is very well captured by the implicit regularization.

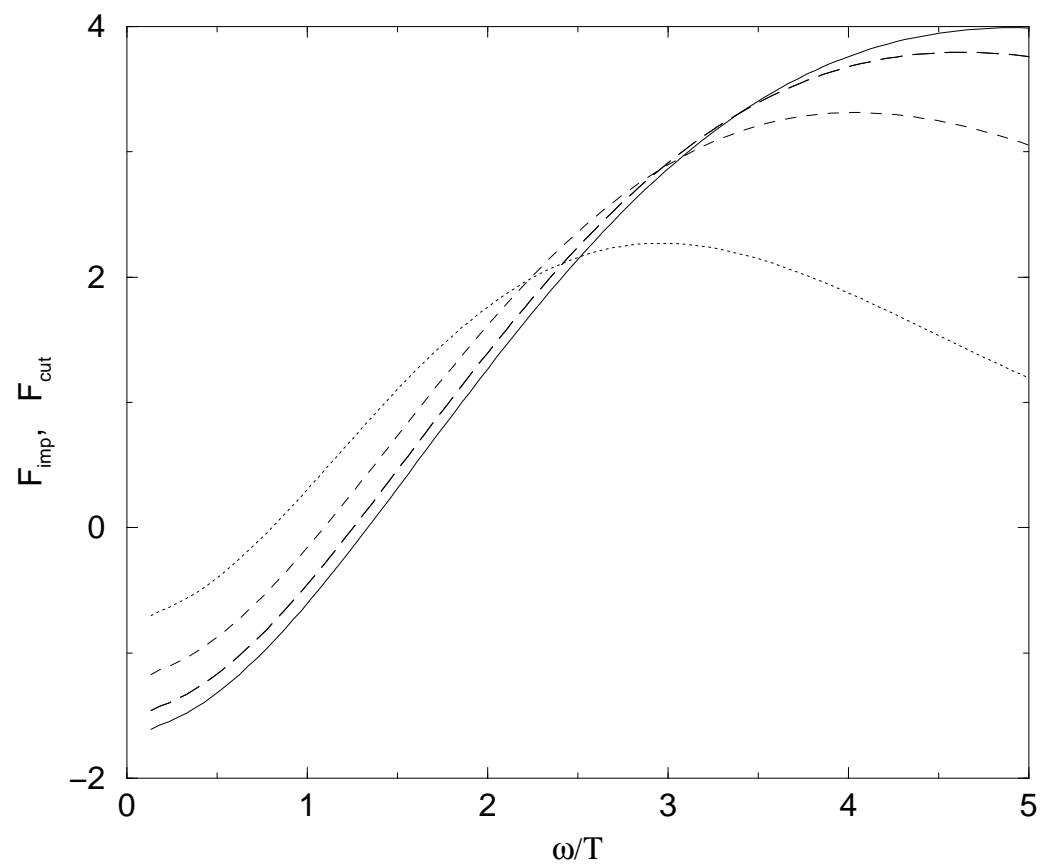

FIG. 1: The functions $F_{i m p}\left(\omega^{2}, T\right)$ (solid line) and $F_{c u t}\left(\omega^{2}, T\right)$ for different values of the cutoff: $\alpha=0.5 \mathrm{GeV}$ (dotted), $\alpha=1.0 \mathrm{GeV}$ (dashed), $\alpha=2.0 \mathrm{GeV}$ (long-dashed).

\section{ACKNOWLEDGMENTS}

Work partially financed by CAPES, CNPq and Fapesp.

[1] R. Casalbuoni, R. Gatto, G. Nardulli and M. Ruggieri., Phys. Rev. D 68, 034024 (2003).

[2] Bing He, Hu Li, C.M. Shakin and Qing Sun, Phys. Rev. C 67, 065203 (2003).

[3] Hu Li, C.M. Shakin and Qing Sun, hep-ph/0310254 
[4] O.A. Battistel and M.C. Nemes, Phys. Rev. D 59, 055010 (1999).

[5] O.A. Battistel and G. Krein, Mod. Phys. Lett. A 18, 2255 (2003).

[6] R.L.S.Farias, G.Krein and O.A. Battistel, in preparation (2004). 